\title{
A 34 years old nulliparous patient with a metastatic mixoild leiomyosarcoma of the uterus : a case report
}

\section{Introduction}

A 34 years old patient underwent a laparoscopic myomectomy for a symptomatic leiomyoma with the final diagnosis of myxoïd leiomyosarcoma.

Uterine sarcomas are rare pathologies accounting for 2-6\% of malignancies of the uterine corpus. The diagnosis is very difficult in the pre-operative work-up, during the surgery and even in the pathological analysis often requiring the opinion of a sarcoma specialist.

There is no consensus or guidelines about risk factors and US-MRI signs for sarcomas. The only risk factor recognized is the age with an increased risk after 55 years old and a virtually zero risk under 35 years old what was the case of our patient.

Furthermore, it is now well known that tumor morcellation worsen the prognosis and increase the risk of recurrence in case of an unknown malignancy of the uterus.

\section{Material and method}

The patient presented for abnormal bleeding during menstruations.

The ultrasonography showed a myoma FIGO $2-5$ of $9 \mathrm{~cm}$ without an abnormal vascularization.

A pelvic MRI was performed without any sign of malignancy (Picture 1).

A laparoscopic myomectomy was performed. The first diagnosis was a smooth muscle tumor of uncertain malignant potential (STUMP). After reevaluation by a specialized pathologist and used of multiple immunohistochemical tests (Fig. 1), the final pathologic diagnosis was a mixoïd leiomyosarcoma. We performed a TEP-SCAN, which showed a slow uptake in the uterine scar without regional or distant metastasis. The TNM classification was pT1b $(7 \mathrm{~cm})$ cNO cM0. The recommendation of our tumor board was to perform a total hysterectomy with ovarian preservation.

\begin{tabular}{|c|c|}
\hline Immunochemistry & Results \\
\hline Desmine & Diffuse positive \\
\hline Caldesmone & Negative \\
\hline Transgeline & Negative \\
\hline ER & Diffuse positive \\
\hline PR & Diffuse positive \\
\hline Cycline D1 & Diffuse positive \\
\hline p53 & Wild Type \\
\hline FH & Guarded expression \\
\hline FISH & Negative \\
\hline Molecular biology & Negative \\
\hline CGH-array & Little overhauled \\
\hline Mitotic index & 1 \\
\hline Number of mitoses & $4 / 10$ HPF \\
\hline Vascular emboli & No \\
\hline Fig. 1. Immunohistochemical tests & \\
\hline
\end{tabular}

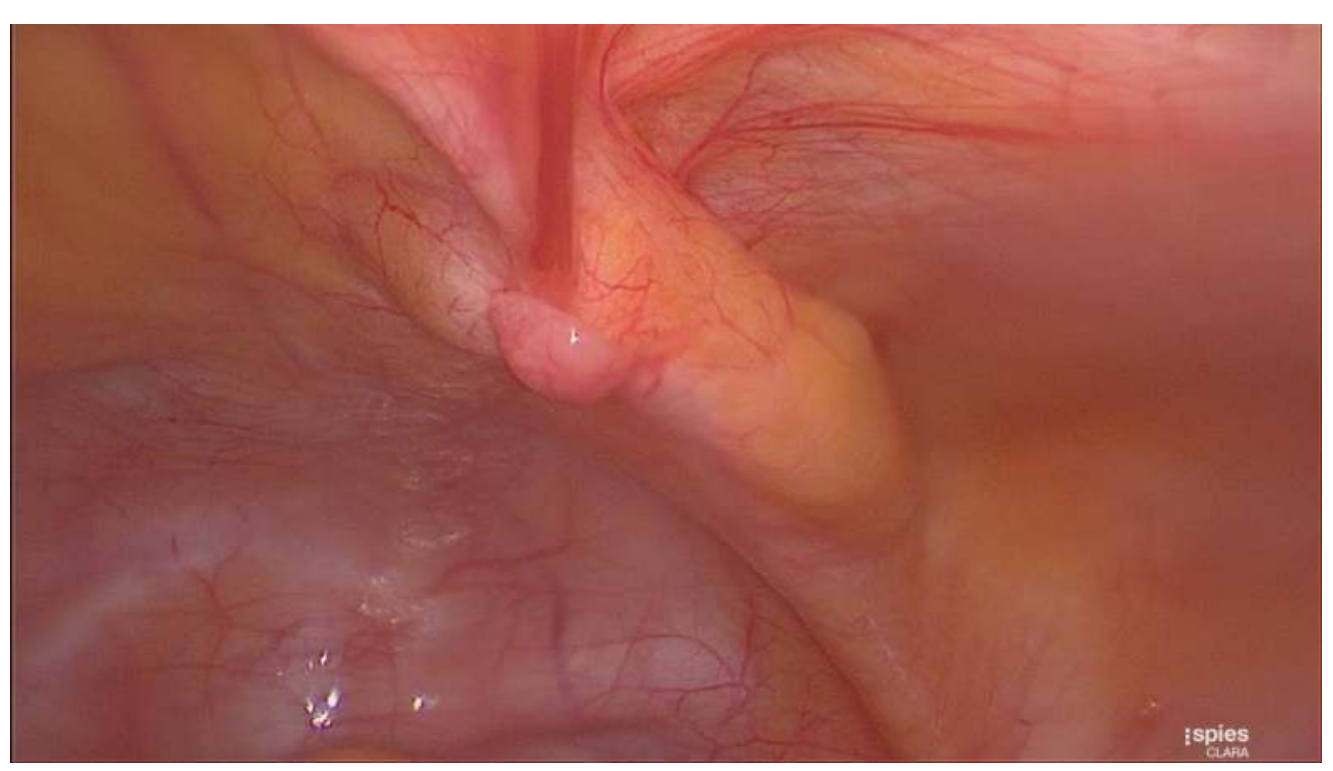

Picture 2. Anterior wall implant

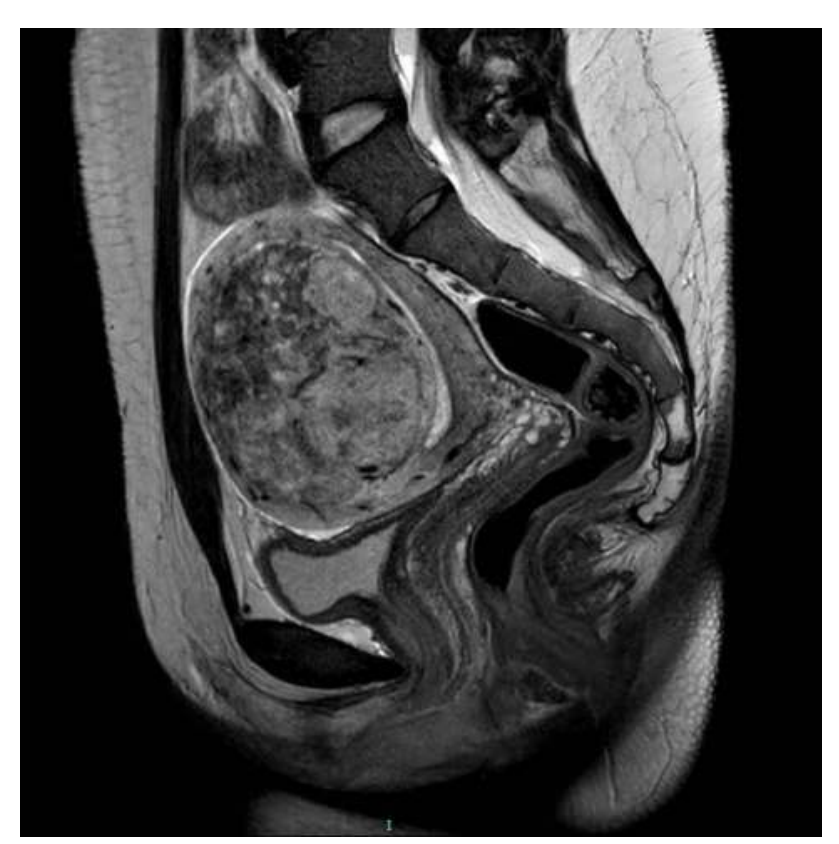

Picture 1. Preoperative MRI, T2 sequence

\section{Results}

An open laparoscopic surgery was performed with a cytology of the peritoneal fluid and a full abdomino-pelvic evaluation finding an implant in the anterior wall in the scar of the sus-pubic trocar (Picture 2). There was no other sign of dissemination. We proceed with an excision of this implant and with a total hysterectomy. A residual zone of myxoïd leiomyosarcoma in the uterus and the abdominal wall was found. The peritoneal cytology showed no tumoral cells.

We asked four specialized centers for the treatment and had three propositions: chemotherapy alone, chemotherapy with radiotherapy and following alone. After discussion with the patient, she decided for a follow-up with a $\mathrm{MRI}$ each 3 months.

Six weeks after surgery, the patient is well without any pain. Three months after surgery, the MRI and the CT-scan are non suspicious.

A second look laparoscopy will be done 6 months later.

\section{Conclusion}

Uterine leiomyosarcoma are rare pathologies with a bad prognosis and a very difficult diagnosis. Usually it occurs in women in the sixth decade but young women are not exempted. Because of the scarcity of these pathology, there is no consensus for the adjuvant treatment. 